Supporting information for

\title{
Switchable Isotropic/anisotropic Wettability and Programmable Droplet Transportation on Shape-memory Honeycomb
}

Liang Zhang, Jingxin Zhao, Jinyan Xu, Jiaqin Zhao, Yutian Zhu, Yongjin Li, Jichun You*

College of Material, Chemistry and Chemical Engineering, Hangzhou Normal University, Hangzhou, 310036, China

*Corresponding author .

E-mail: you@hznu.edu.cn 


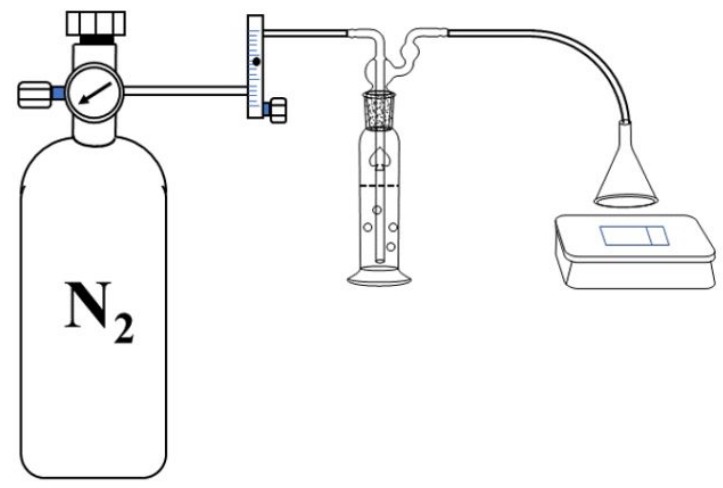

Fig. S1. Home-made setup for the fabrication of PLLA honeycombs by means of breath figure.

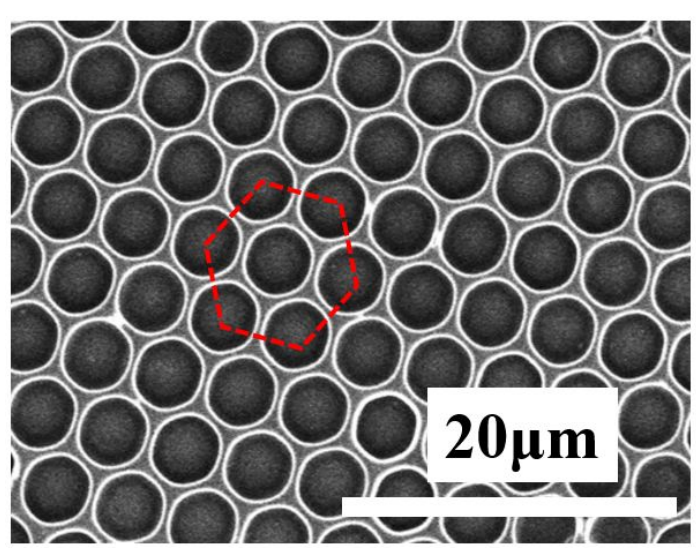

Fig. S2. Typical SEM images of PLLA honeycomb and HCP pores on it (red dash lines).

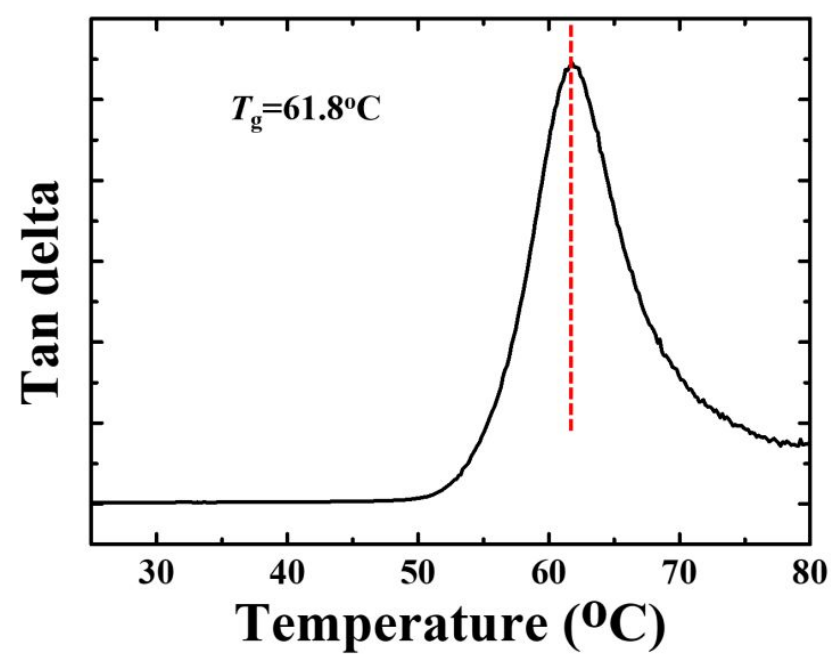

Fig. S3. DMA Tan delta curve of PLLA honeycomb. 


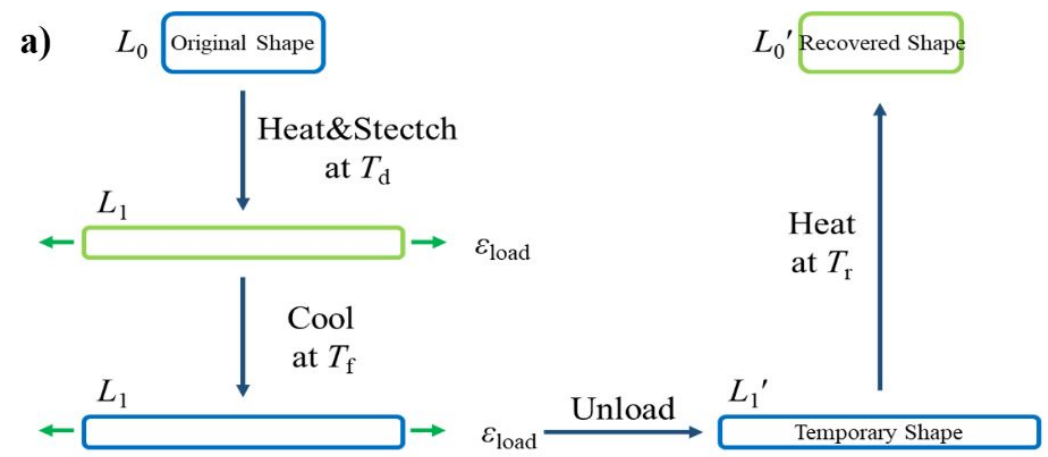

b)

Shape fixity $=\left(L_{1}^{\prime}-L_{0} / L_{1}-L_{0}\right) \times 100 \%$; Recovery ratio $=\left(L_{1}^{\prime}-L_{0}^{\prime} / L_{1}^{\prime}-L_{0}\right) \times 100 \%$

Fig. S4. Schematic description of the shape memory testing by means of tension method (a) and the calculation equations (b) of shape fixity and recovery ratio.

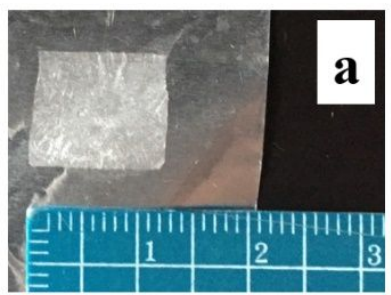

$\mathrm{DR}=1$

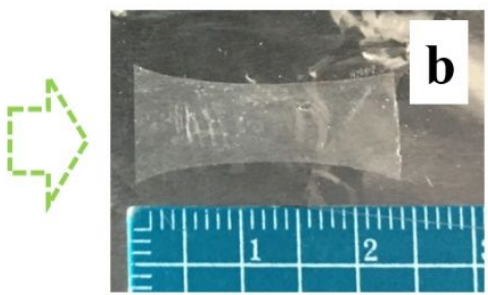

$\mathrm{DR}=2.4$

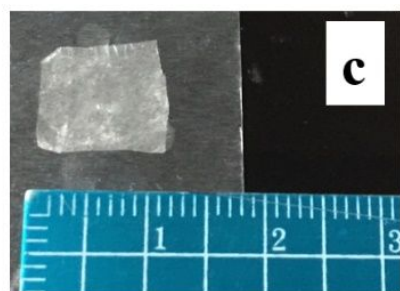

$\mathrm{DR}=1$

Fig. S5. A typical shape memory cycle with the indicated draw ratio $\mathrm{DR}=1$ (a, before uniaxial tension), $\mathrm{DR}=2.4$ ( $\mathrm{b}$, after tension) and $\mathrm{DR}=1$ ( $\mathrm{c}$, recovery to permanent shape).

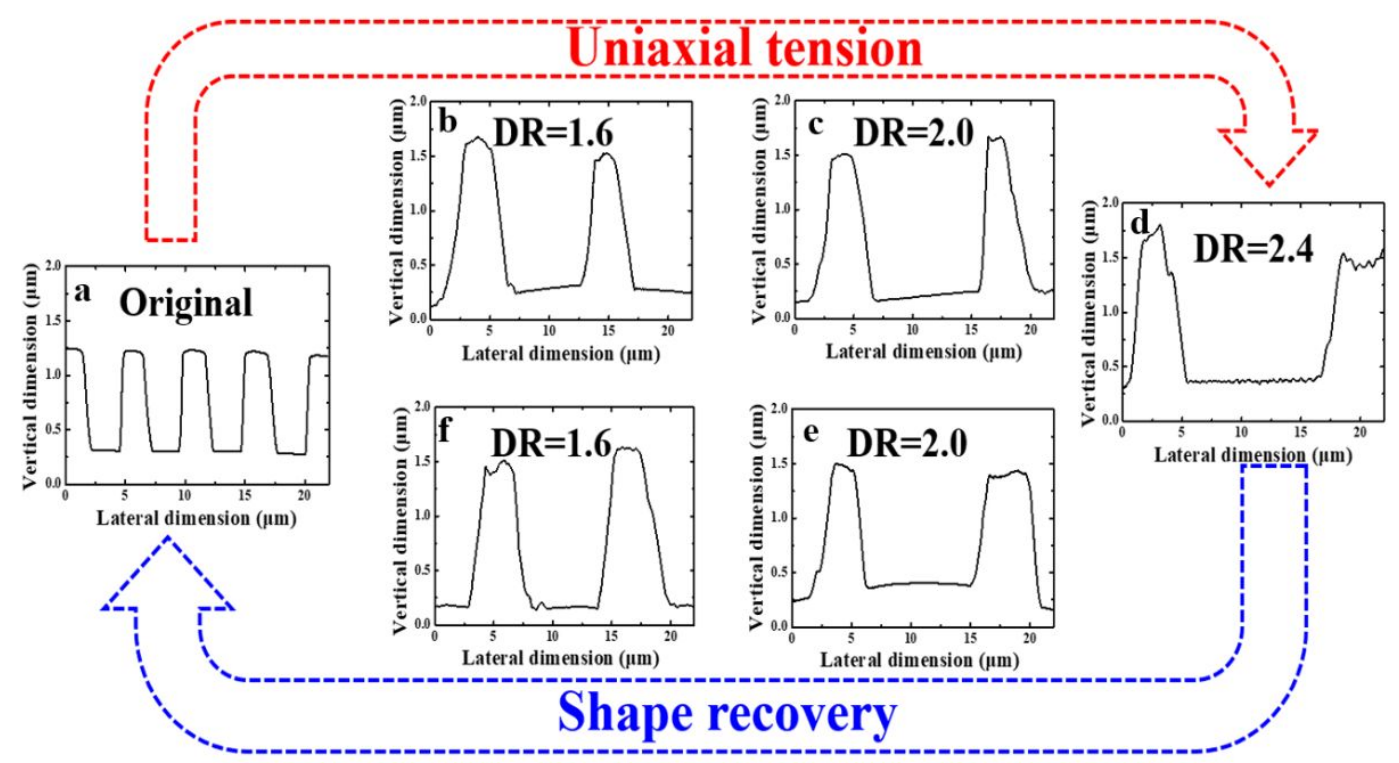


Fig. S6. The line-cut profiles in AFM images during the shape memory and recovery processes. (a) before uniaxial tension, (b) $\mathrm{DR}=1.6$, (c) $\mathrm{DR}=2.0$, (d) $\mathrm{DR}=2.4$, (e, f) Draw ratio of uniaxial stretch back to 2.0 and 1.6 respective.
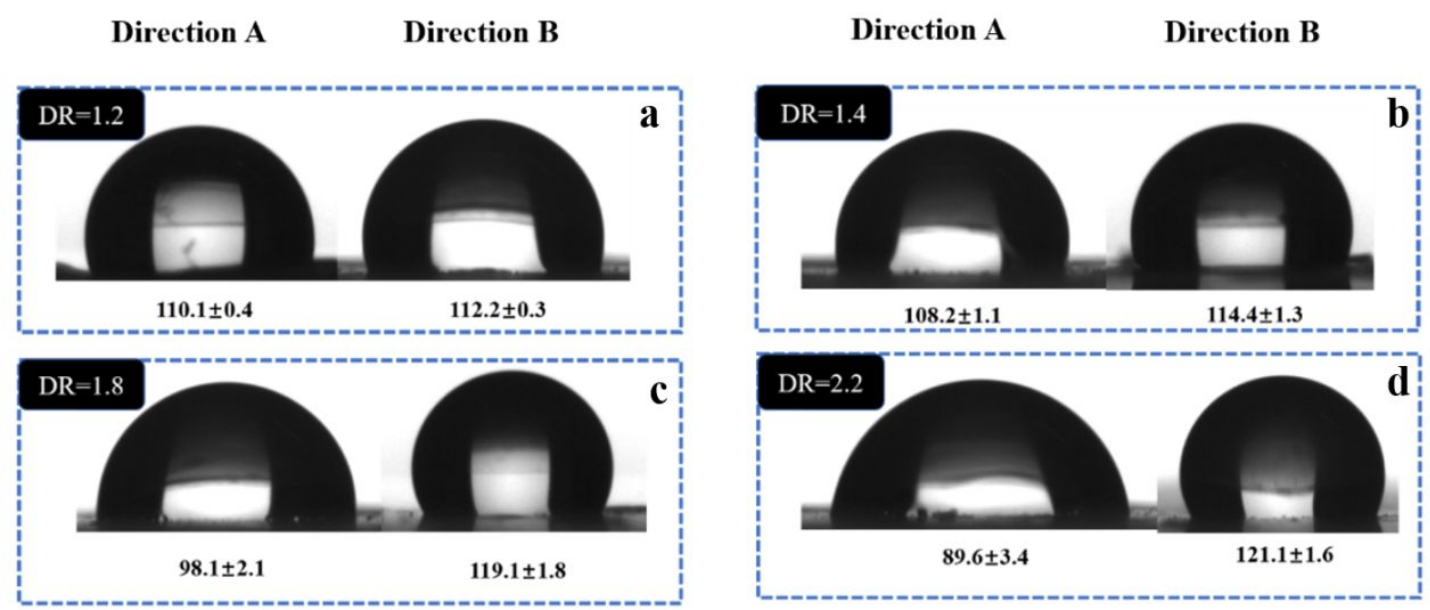

Fig. S7. Contact angles in direction $\mathrm{A} / \mathrm{B}$ on specimens with the indicated draw ratios (a) $\mathrm{DR}=1.2$,

( b ) $\mathrm{DR}=1.4$, (c) $\mathrm{DR}=1.8$, (d) $\mathrm{DR}=2.2$.

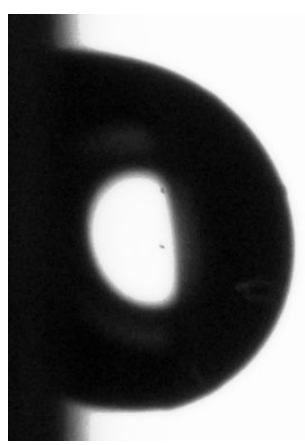

Fig. S8. a typical example, even the specimen with draw ratio of 2.4 (direction A) exhibits the sliding angle of $90^{\circ}$. On all PLLA honeycomb surfaces, water droplets were pinned (data not shown here) and cannot roll down.

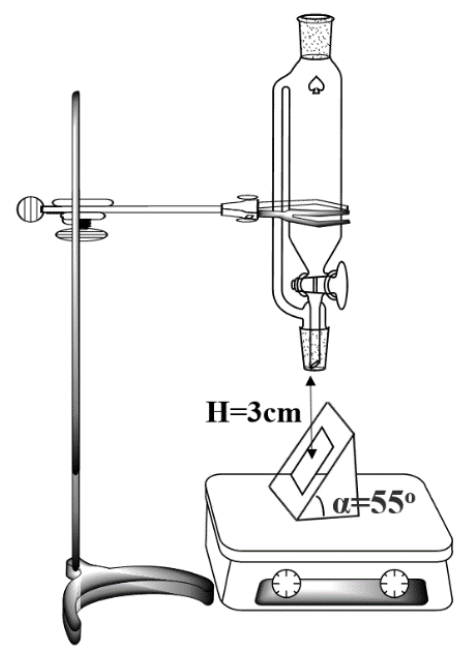


Fig. S9. Home-made setup for the droplet transportation on PLLA honeycomb. The height and tilted angle are $3 \mathrm{~cm}$ and $55^{\circ}$ respectively.

a)

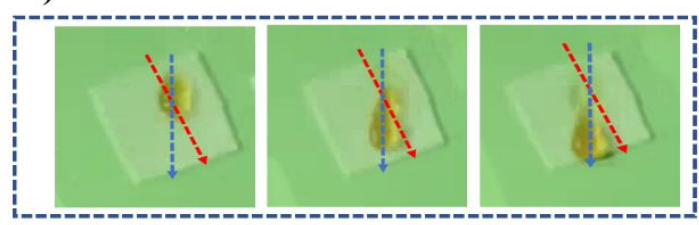

c)

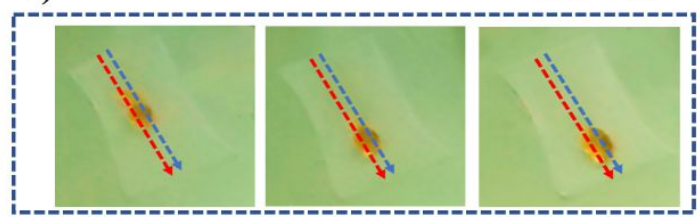

b)

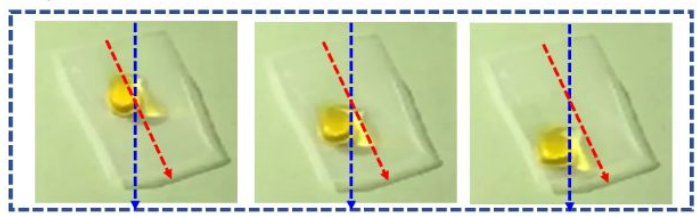

d)

$\mathrm{DR}=2.4$

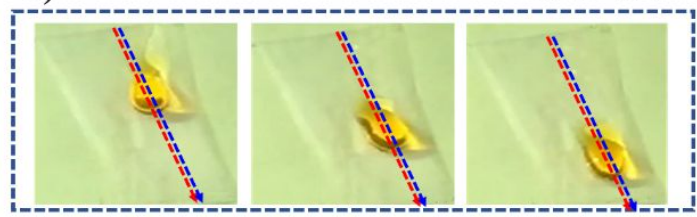

Fig. S10. The movement of water droplet on PLLA honeycomb with various draw ratio of 1.2(a), 1.6(b), 2.0(c) and 2.4(d) draw ratios. The red and blue lines represent the oriented pores direction and droplet movement direction respectively.

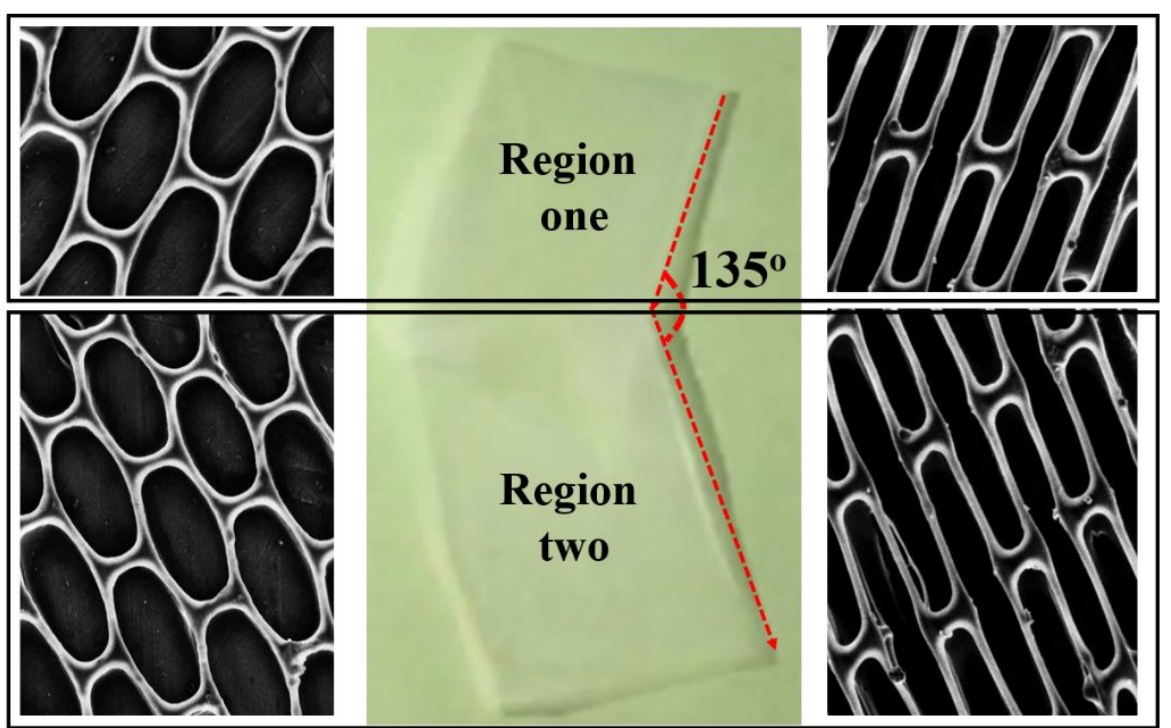

$\mathrm{DR}=1.6$

$\mathrm{DR}=2.4$

Fig. S11. The details of V-shape specimen with two regions exhibiting different oriented pores illustrated by SEM images.

Movies 1-4 display the water droplet transportation on the simple (Movie 1 and 2) and V-shape 
(Movie 3 and 4) PLLA honeycomb surface with the draw ratio of 1.6 (Movie 1 and 3) and 2.4 (Movie 2 and 4). The movies are shown at playing speed $* 6$. 